\title{
Core Loss Prediction in Electrical Machine Laminations Considering Skin Effect and Minor Hysteresis Loops
}

\author{
Maged Ibrahim, Member, IEEE, Pragasen Pillay, fellow IEEE
}

\begin{abstract}
This paper presents a method for the estimation of core losses in electrical machine laminations exposed to high frequency and non-sinusoidal excitations by using only low frequency measurements. The developed model takes into account the non-uniform distribution of the magnetic field inside the lamination. Accurate core loss prediction in the presence of minor loops is achieved using the Energetic model to calculate the quasi-static hysteresis loops. The results are verified experimentally by comparing to the measured core losses in laminations exposed to the flux waveforms in different sections of an inset permanent magnet (PM) machine. The comparison between measured and calculated core losses shows excellent agreement, confirming the validity of the model.
\end{abstract}

Index Terms - Core loss, Eddy current loss, Hysteresis loss, Skin effect, Minor loops.

\section{INTRODUCTION}

Core losses in electrical machines account for a large portion of the total losses ranging from $15-25 \%$ in induction machines operating with sinusoidal supplies [1] and even higher for permanent magnet (PM) machines and switched reluctance (SR) machines. Improving the machine efficiency by design optimization requires accurate quantification of core losses during the machine design stage.

In practice, lamination manufacturers usually provide core loss data under sinusoidal excitations in a limited frequency and flux density range. This data is not adequate for the prediction of core losses in high speed electrical machines which require loss information at high frequencies and high flux densities. In addition, the actual flux waveforms inside many machines, e.g., PM machines and SR machines are nonsinusoidal and differ in different parts of the machine. The flux distortion can also be caused by non-sinusoidal supplies, e.g., pulse width modulation (PWM) inverter fed induction

Manuscript received October 1st, 2012.

M. Ibrahim is with the Department of Electrical and Computer Engineering, Concordia University, Montreal, QC, Canada, (e-mail: ma_ib@encs.concordia.ca).

P. Pillay is with the Department of Electrical and Computer Engineering, Concordia University, Montreal, QC, Canada, and also an adjunct Professor at the University of Cape Town, Cape Town, South Africa (e-mail: pillay@encs.concordia.ca). machines. Since there are an infinite number of possible encountered flux waveforms in electrical machines, it is difficult to accurately predict the machine core losses using curve fitting techniques that utilize sinusoidal core loss data. Therefore, core loss prediction under non-sinusoidal flux should be performed using a physics-based core loss model that is derived from the physical characteristics of the core loss mechanism in magnetic materials, and can describe the non-linear nature of core losses under distorted flux.

Most of the earlier work on core loss modeling was done by Steinmetz [2]. In his work, the core loss in a magnetic material is considered the addition of hysteresis and eddy current losses. The total core loss is represented by,

$P=K_{h} f B^{n}+K_{e} f^{2} B^{2}$

$K_{h}$ and $K_{e}$ are the hysteresis and eddy current loss coefficients, which can be extracted from the measured data, and $n$ is a Steinmetz constant equal to 1.6. The Steinmetz constant $n$ is found to be dependent on the material type, as well as the flux density. In addition, an analytical solution of Maxwell's equations with an assumption of uniform magnetic field distribution, allows the eddy current coefficient $K_{e}$ to be expressed as function of the material electrical conductivity $\sigma$ and the lamination thickness $2 L$ as,

$K_{e}=\frac{(2 L)^{2} \pi^{2} \sigma}{6}$

The eddy current loss with the loss coefficient $K_{e}$ calculated by (2) is known as the classical eddy current loss. It was found that the measured eddy current losses are higher than the calculated classical eddy current losses. The difference is known as the excess losses. Based on statistical loss theory, Bertotti [3] proposed an additional term to account for the excess losses. Therefore, the Steinmetz core loss formula was modified to,

$P=K_{h} f B^{n}+K_{e} f^{2} B^{2}+K_{e x} f^{1.5} B^{1.5}$

where $K_{e x}$ is dependent on the material micro-structure, the conductivity, and the cross sectional area of the lamination. The coefficients of the three-term formulation are generally obtained from the measured core loss data. However, the calculated losses from (3) are only accurate within a certain frequency and flux density range. In more recently developed models [4, 5], this range is extended by allowing the coefficients to vary with the frequency and the flux density. 
However, the determination of these variable coefficients requires additional core loss data at high frequencies and high flux densities.

In this paper, a physics based core loss model is developed to calculate core losses in electrical machine laminations exposed to high frequency and non-sinusoidal flux using low frequency core loss measurements. Section I presents an analytical core loss model that can calculate the losses in machine laminations considering the non-uniform distribution of the magnetic field inside the lamination. The model results are then compared to the measured losses in laminations exposed to the flux waveforms in different sections of a PM machine. Section II presents a fast and accurate method for calculating minor hysteresis loop losses by using the energetic model to predict the material static magnetization behavior under any arbitrary flux waveform.

\section{CORE LOSS MODELING IN THE ABSENCE OF MINOR HYSTERESIS LOOPS}

Most core loss models are based on the assumption of uniform magnetic field distribution across the lamination. While these models can achieve satisfactory accuracy of core loss prediction at power frequencies, there are large discrepancies between measured and calculated losses at high frequencies. This deviation is mainly attributed to the influence of skin effect on core loss components. When the laminations are exposed to time varying magnetic fields, eddy currents are induced in the laminations; these currents produce a secondary magnetic field that opposes the applied field. The field created by the eddy currents is maximum at the lamination center, where the contribution of eddy currents adds, and minimum at the lamination surface. Therefore, the total magnetic field becomes non-uniform across the lamination thickness, as the field magnitude at the lamination surface is higher than its magnitude at the center of the lamination. This phenomenon (skin effect) is pronounced when the lamination is exposed to high frequency excitations, especially for thicker laminations. Accurate core loss calculation in the presence of skin effect can be achieved using numerical models [21-23]. However, these approaches are not suitable for electrical machine design and optimization, where a computationally efficient method is required, as the core loss calculation has to be performed in each machine mesh element. Hence, analytical core loss models are still the preferred choice for electrical machine design.

\section{A. Eddy current loss}

The confinement of the magnetic field to the lamination surface due to skin effect changes the effective resistance of the eddy current path, as the currents are forced to circulate through a smaller area, which increases the resistance of the eddy current path, and therefore decreases the eddy current losses in the lamination. This explains why the eddy current losses calculated assuming a uniform magnetic field distribution overestimates the losses at high frequencies.

Many analytical models are presented in the literature to calculate the eddy current loss in electrical machine laminations taking into account the non-uniform magnetic field distribution inside the lamination. The eddy current losses calculated using the material properties and the lamination dimensions are much lower than the total measured eddy current losses. The difference is usually compensated for by extracting the coefficient from the experimental data [6], or by using variable core loss coefficients that requires using high frequency core loss measurements [7]. The main reason for this divergence stems from the fact that the developed formulas express the eddy current loss as a function of the flux density at the boundary, which differs from the measured average flux density over the lamination when skin effect is significant. In [8] an analytical solution of Maxwell's equations assuming a linear magnetic material allows the peak average flux density $B_{a v}$ to be expressed as a function of the peak flux density at the boundary $B_{b}$. The eddy current loss can then be represented as a function $B_{a v}$ as,

$$
P_{e}=\frac{\omega B_{a v}^{2}}{2 \lambda \mu \rho} \frac{\left[\exp \left(\frac{4}{\lambda}\right)+2 \exp \left(\frac{2}{\lambda}\right) \cos \left(\frac{2}{\lambda}\right)+1\right]\left[\exp \left(\frac{4}{\lambda}\right)-2 \exp \left(\frac{2}{\lambda}\right) \sin \left(\frac{2}{\lambda}\right)-1\right]}{\left[1-2 \exp \left(\frac{4}{\lambda}\right) \cos \left(\frac{4}{\lambda}\right)+\exp \left(\frac{8}{\lambda}\right)\right]}
$$

where $\lambda=\sqrt{\frac{2}{L^{2} \omega \mu \sigma}}, \omega, \rho$ and $\mu$ are the angular frequency, the mass density and the magnetic permeability.

In order to ensure that skin effect is pronounced, the calculated loss by (4) is compared to the measured eddy current loss in M19 non-oriented steel lamination with a thickness of $0.635 \mathrm{~mm}$ at $400 \mathrm{~Hz}$. It is observed that the eddy current loss calculated by (4) underestimates the loss, especially at high flux densities. This divergence is mainly attributed to the assumption of constant magnetic permeability. This assumption implies that the flux density at any position in the lamination is linearly related to the field intensity. While this allows an analytical solution of Maxwell's equation, the actual differential permeability of the material is varying both in time and space. Fig. 1 shows the flux density variation. It can be seen that, due to skin effect, the flux density waveforms differ in magnitude and phase along the lamination thickness. This causes different hysteresis loops to occur at different points inside the lamination. With the differential permeability defined as the local slope of the hysteresis loop, the permeability is changing along the lamination thickness with the hysteresis loop variation. In addition, at a certain position inside the lamination, the differential permeability is also changing in time, as the local slope of the hysteresis loop is varying during the electrical cycle, being maximum at coercivity, and decreasing towards the tip of the hysteresis loop. Also, the magnetic permeability is affected by the value of the peak measured average flux density, as the local hysteresis loop shape is strongly dependent on the flux density level. The choice of the effective magnetic permeability is a critical factor in the determination of the eddy current losses, as its value controls the magnetic field distribution inside the lamination. It is observed that skin effect increases for higher 
values of permeability. The decrease in the effective area of the magnetic field due to a higher permeability increases the resistance of the eddy currents path, and decreases the eddy current loss calculated by (4) for the same peak average flux density. Higher losses are reported in [8] by choosing the effective permeability according to the value of the magnetic field at the boundary. The calculated losses are higher than the measurements because the value of magnetic permeability substituted in Maxwell's equation describes the relationship between the flux density and the field intensity at any position inside the lamination. At higher frequencies, the magnetic field inside the lamination is much lower than the field at the boundary, due to skin effect. Therefore, the magnetic permeability chosen according to the field at the boundary does not reflect the permeability variation inside the lamination, hence the overestimation of the eddy current loss. In order to account for the variation of the magnetic permeability through the lamination, the permeability is chosen according to the average flux density over the lamination, this parameter can be easily obtained from experiment. The magnetic permeability variation in time is approximated by considering the slope of the line from the origin of the hysteresis loop to the peak flux density. The permeability that represents the material magnetic behavior is obtained from low frequency measurements, where the magnetic field is uniform through the lamination. This permeability is expressed as a function of the peak average flux density $B_{a v}$ with a $4^{\text {th }}$ order polynomial,

$\mu\left(B_{a v}\right)=K_{0}+K_{1} B_{a v}+K_{2} B_{a v}{ }^{2}+K_{3} B_{a v}{ }^{3}+K_{4} B_{a v}{ }^{4}$

Therefore, $\lambda$ is modified to $\lambda\left(B_{a v}\right)=\sqrt{\frac{2}{L^{2} \omega \sigma \mu\left(B_{a v}\right)}}$, and the eddy current loss is expressed as,

$$
\begin{aligned}
& P_{e v p}=\frac{\omega K_{E} B_{a v}^{2}}{2 \lambda \mu \rho}\left[1-2 \exp \left(\frac{4}{\lambda\left(B_{a v}\right)}\right) \cos \left(\frac{4}{\lambda\left(B_{a v}\right)}\right)+\exp \left(\frac{8}{\lambda\left(B_{a v}\right)}\right)\right]^{-1} \\
& \times\left[\exp \left(\frac{4}{\lambda\left(B_{a v}\right)}\right)+2 \exp \left(\frac{2}{\lambda\left(B_{a v}\right)}\right) \cos \left(\frac{2}{\lambda\left(B_{a v}\right)}\right)+1\right] \\
& \times\left[\exp \left(\frac{4}{\lambda\left(B_{a v}\right)}\right)-2 \exp \left(\frac{2}{\lambda\left(B_{a v}\right)}\right) \sin \left(\frac{2}{\lambda\left(B_{a v}\right)}\right)-1\right]
\end{aligned}
$$

The coefficient $K_{E}$ can be determined using core loss measurements at power frequencies by simply dividing the separated eddy current loss at one peak flux density value by the classical eddy current loss.

Fig. 2 compares the eddy current loss calculated by (6) with the measured eddy current loss at $400 \mathrm{~Hz}$. It is clear that the formula predicts accurately high frequency eddy current losses using only low frequency core loss data. It also can be seen that (1) overestimates the eddy current loss at high frequency. Also, the addition of classical and excess eddy current losses calculated by (3) is much lower than the measured eddy current loss at high frequency and high flux densities.

For any particular flux waveform with a fundamental frequency $f_{1}$, the flux density is decomposed into a Fourier series of harmonics. The contribution of each component into

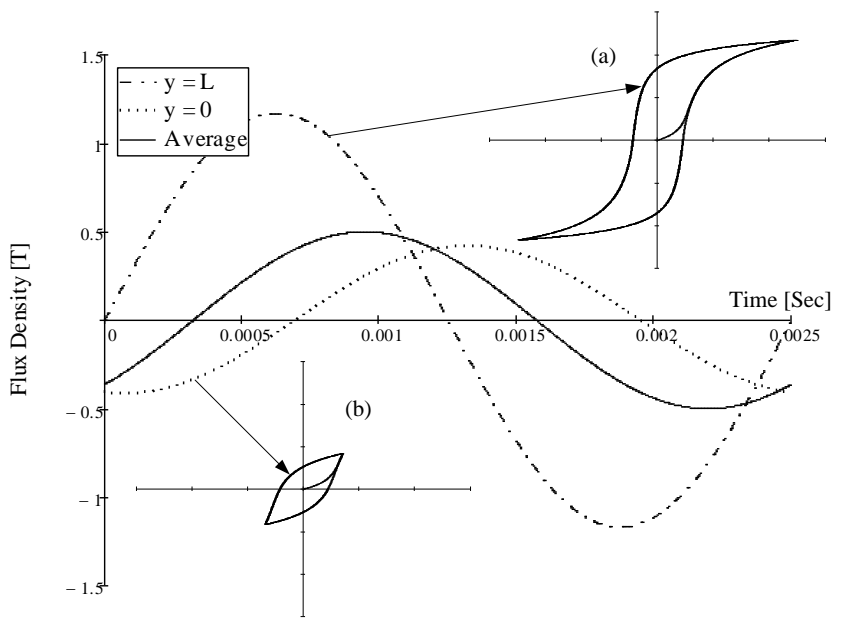

Fig. 1. Illustration of flux density variation in time and space for M19G24 at $400 \mathrm{~Hz}$ with (a) and (b) being the simulated hysteresis loops at the surface and center of the lamination, respectively.

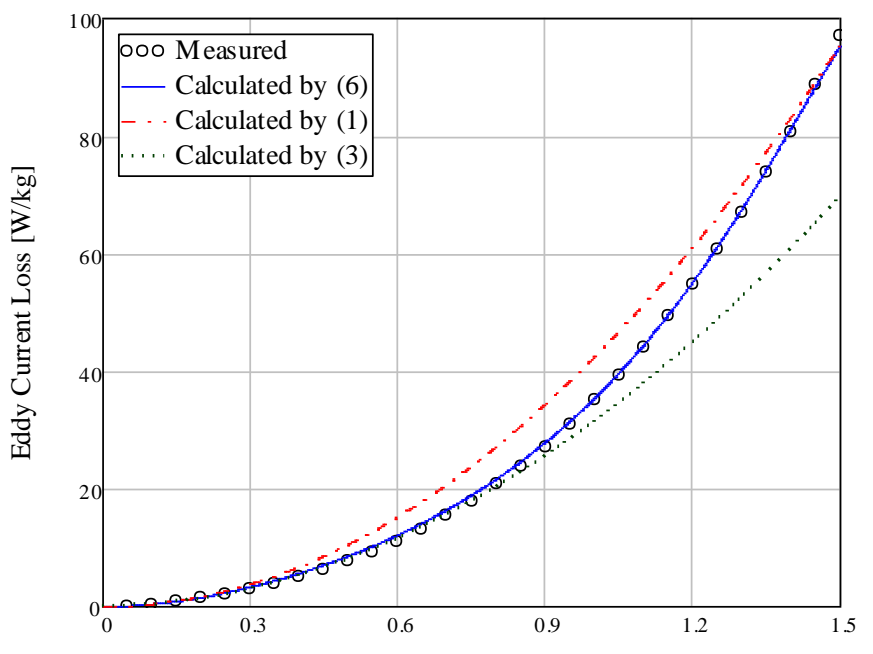

Flux Density [T]

Fig. 2. Comparison of the calculated eddy current losses by different formulas with the measured losses for M19G24 at $400 \mathrm{~Hz}$.

the eddy current loss is calculated based on the magnitude of the harmonic, and the harmonic frequency which determines the flux penetration of the individual harmonics. The total eddy current loss is then calculated by,

$$
P_{e T}=\sum_{i=1}^{N} P_{e v p}\left(B_{i}, i f_{1}\right)
$$

\section{B. Hysteresis loss}

The hysteresis energy loss is generally assumed independent of the frequency. This assumption is valid for low frequency excitations where the magnetic field distribution is uniform inside the lamination. On the other hand, when the lamination is subject to high frequency excitation, the skin effect causes the peak flux density to vary in different parts of the lamination; this causes the local hysteresis loops, and therefore the local hysteresis energy loss per cycle to differ at different points inside the lamination. The hysteresis loss at high frequencies can be calculated by constructing the magnetic field distribution across the lamination. However 
this procedure is computationally expensive, especially when the flux density waveform is non-sinusoidal, as it becomes difficult to obtain the flux density distribution using analytical models. Therefore, this method is not suitable for core loss determination in finite element (FE) machine design software, which requires fast core loss calculations at each mesh element. Therefore, the total hysteresis energy loss is assumed to be only dependent on the flux density amplitude. Firstly, the static hysteresis energy loss is separated by extrapolating core loss per cycle vs. frequency curves for different values of flux densities to zero frequency. The energy loss at zero frequency is considered the static hysteresis energy loss per cycle. The separated hysteresis loss at four values of flux density are then used to determine the hysteresis loss coefficients $K_{h}, a, b$ and $c$ [4]. The hysteresis loss is then calculated by,

$P_{h}=f_{1} K_{h} \hat{B}^{a+b \hat{B}+c \hat{B}^{2}}$

The hysteresis loss calculated by (8) is only accurate when there are no minor loops present in the main hysteresis loop. Accurate prediction of hysteresis losses with minor loops is achieved by the method presented in section III.

\section{Experimental verification}

The flux waveforms in different parts of a PM machine core are obtained by FE simulation. These waveforms are then generated in the Epstein frame laminations using the experimental setup described in [9].

The machine under consideration is a 4 pole 24 slot PM machine with inset magnets, designed for use as a traction motor in a lift truck application [10]. The FE simulation is performed for several rotor positions in order to construct the flux density waveforms during one electrical cycle.

The flux waveforms in the stator tooth and the stator yoke of the machine at no load and full load are shown in Fig. 3 and 4 , respectively. These waveforms are then generated in the Epstein frame laminations. The measured losses with these waveforms are shown in Fig. 5. It can be seen that the specific core losses measured using the PM machine flux waveforms at no-load are higher than the losses measured with sinusoidal flux at the same frequency. Also, the stator tooth flux waveform at no-load generates higher losses per $\mathrm{kg}$ of steel than the stator yoke flux. From the measured losses with the flux waveforms at full load, it can be seen that there is a significant increase in the losses generated by the stator tooth flux, which is much higher than the stator yoke losses.

The Fourier series analysis is applied to the four flux waveforms. The harmonic ratios are shown in Table I. It can be observed that there is high harmonic content in the stator tooth flux at no-load, with the 5th harmonic being $15 \%$ of the flux waveform peak. The harmonic content in the stator tooth flux increases when the machine is operating at full load, as the $3 \mathrm{rd}$ and the 5th harmonics become $31 \%$ and $15 \%$ of the waveform peak, respectively.

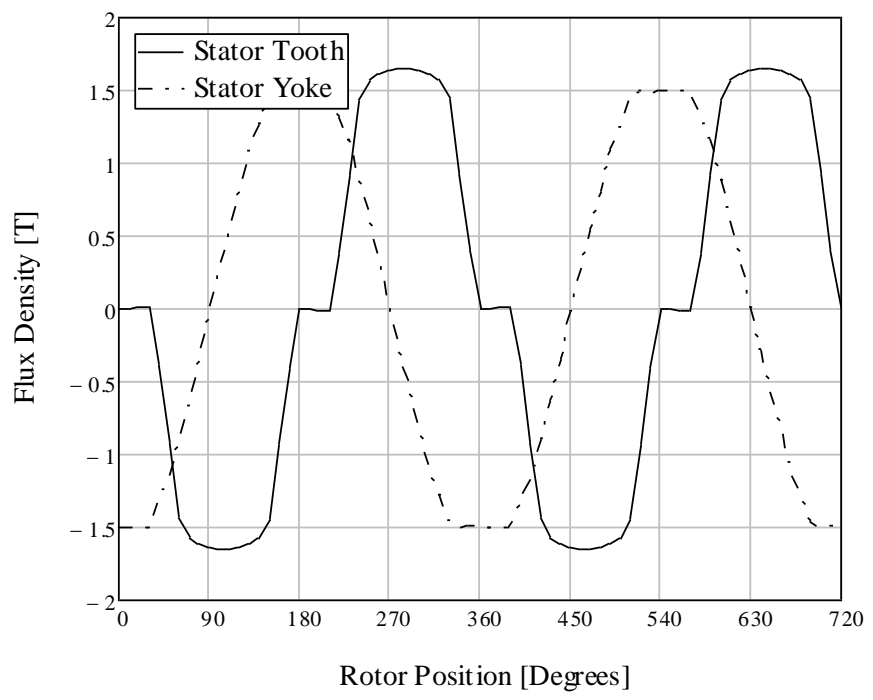

Fig. 3. Flux density waveforms in the stator tooth and the stator yoke of the inset PM machine at no-load.

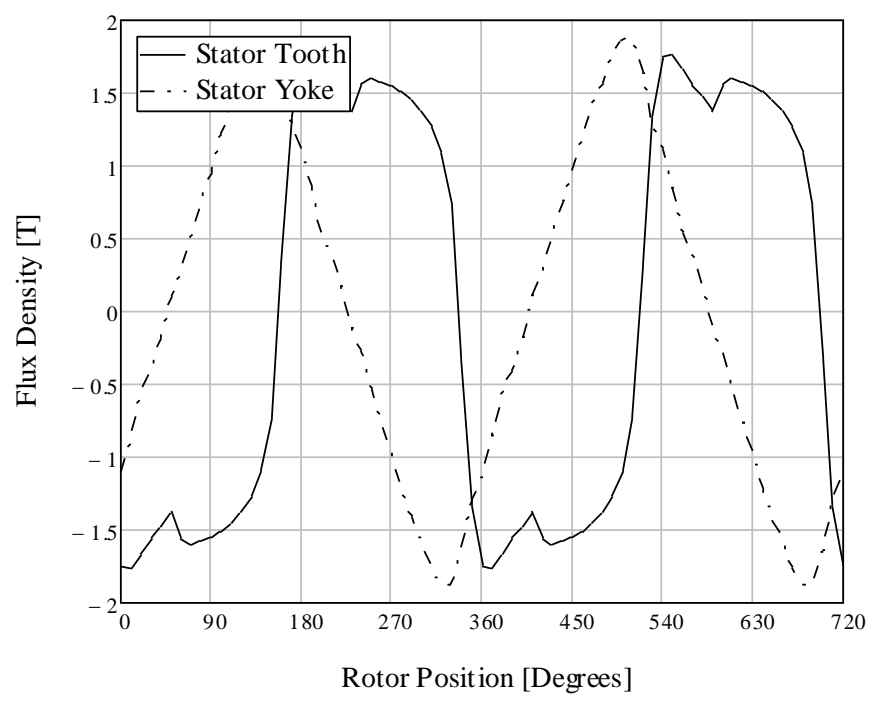

Fig. 4. Flux density waveforms in the stator tooth and the stator yoke of the inset PM machine at full-load.

TABLE I

Flux Density Harmonic Ratios in the Inset PM Machine

\begin{tabular}{|c|c|c|c|c|}
\hline \multirow{2}{*}{$\begin{array}{c}\text { Harmonic } \\
\text { Order (h) }\end{array}$} & \multicolumn{4}{|c|}{$\frac{B_{h}}{B_{p}}$} \\
\cline { 2 - 5 } & $\begin{array}{c}\text { Stator Tooth } \\
\text { No-load }\end{array}$ & $\begin{array}{c}\text { Stator Yoke } \\
\text { No-load }\end{array}$ & $\begin{array}{c}\text { Stator Tooth } \\
\text { Full-load }\end{array}$ & $\begin{array}{c}\text { Stator Yoke } \\
\text { Full-load }\end{array}$ \\
\hline 1 & 1.035 & 1.080 & 1.082 & 0.878 \\
\hline 3 & 0.061 & 0.050 & 0.308 & 0.091 \\
\hline 5 & 0.152 & 0.027 & 0.151 & 0.027 \\
\hline 7 & 0.056 & 0.011 & 0.051 & 0.012 \\
\hline 9 & 0.007 & 0.001 & 0.036 & 0.010 \\
\hline 11 & 0.007 & 0.000 & 0.010 & 0.009 \\
\hline 13 & 0.002 & 0.007 & 0.010 & 0.010 \\
\hline
\end{tabular}


Core losses are then calculated using the proposed core loss model. The total loss is considered the addition of the hysteresis loss calculated by (8) and the eddy current loss calculated by (7). The extracted coefficients for M19G24 steel are listed in table II. The first 13th harmonics are used for the eddy current loss determination. Figs. 6-9 compare the calculated losses with the measured losses for the four flux waveforms in Figs. 3 and 4, when the machine is running at $1912 \mathrm{rpm}$. It is clear that the calculated losses are in excellent agreement with the measured losses for all the flux waveforms. It should be noted that the calculated losses in Fig. 9 do not include the minor loop hysteresis losses generated by the stator tooth flux in Fig. 4, as these losses are found to be of negligible effect on the total losses.

TABLE II

Extracted coefficients for M19G24 steel

\begin{tabular}{|c|c|c|c|c|}
\hline$K_{E}$ & $K_{h}$ & $a$ & $b$ & $c$ \\
\hline 1.497 & 0.014 & 2.214 & -1.415 & 1.015 \\
\hline
\end{tabular}

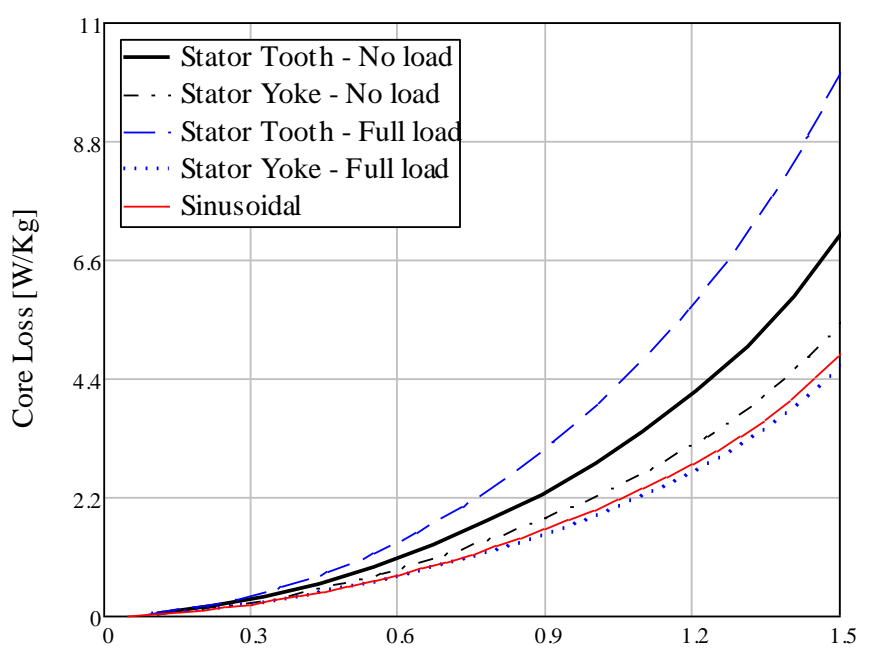

Peak Flux Density [T]

Fig. 5. Comparison of the measured core losses with the flux waveforms the PM machine core at no-load and full-load when operating at $1912 \mathrm{rpm}$.

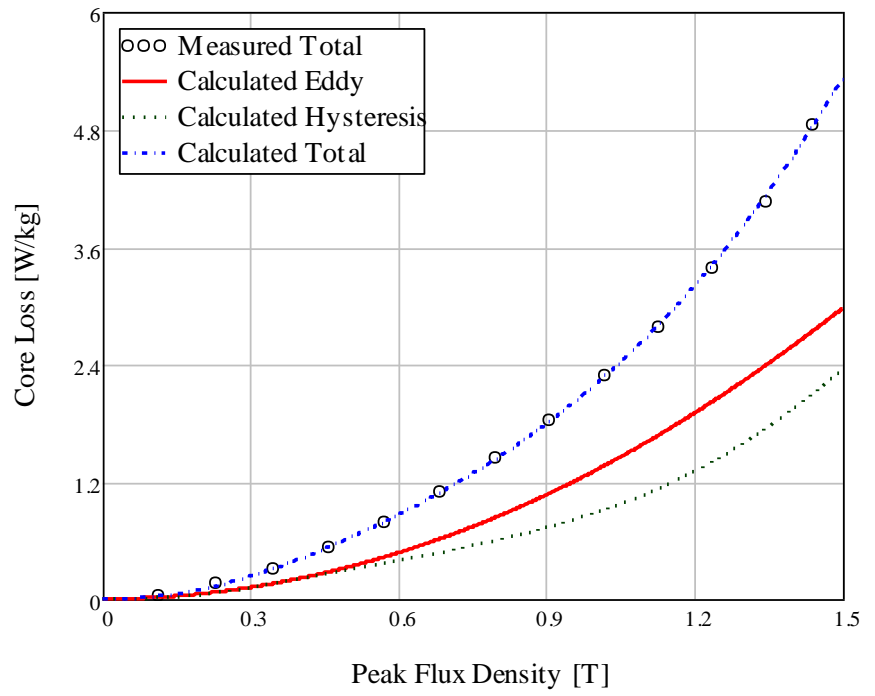

Fig. 6. Comparison of calculated and measured core losses for the flux waveform in the stator yoke at no-load.

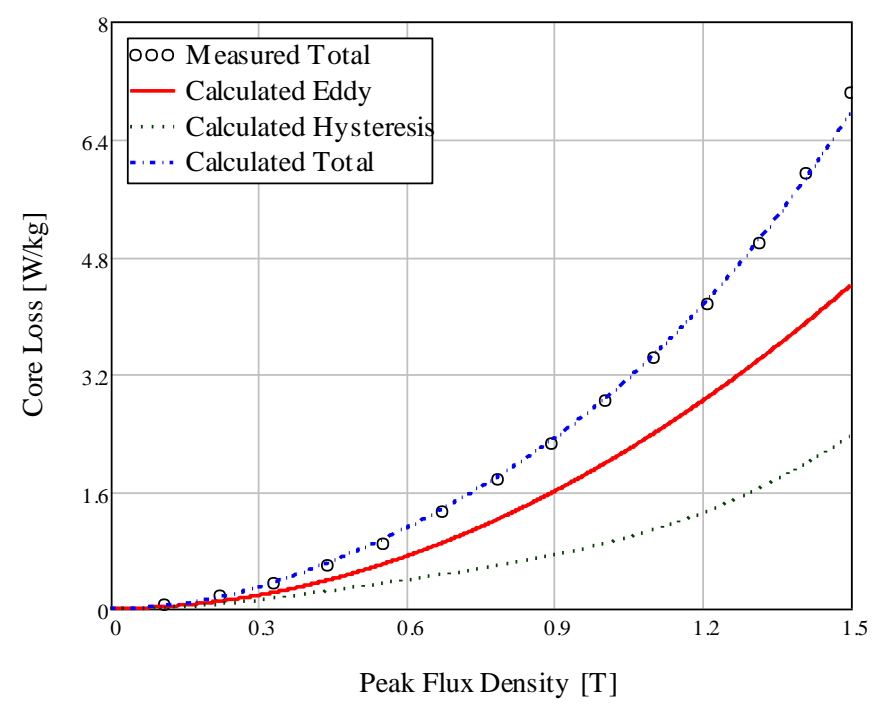

Fig. 7. Comparison of calculated and measured core losses for the flux waveform in the stator tooth at no-load.

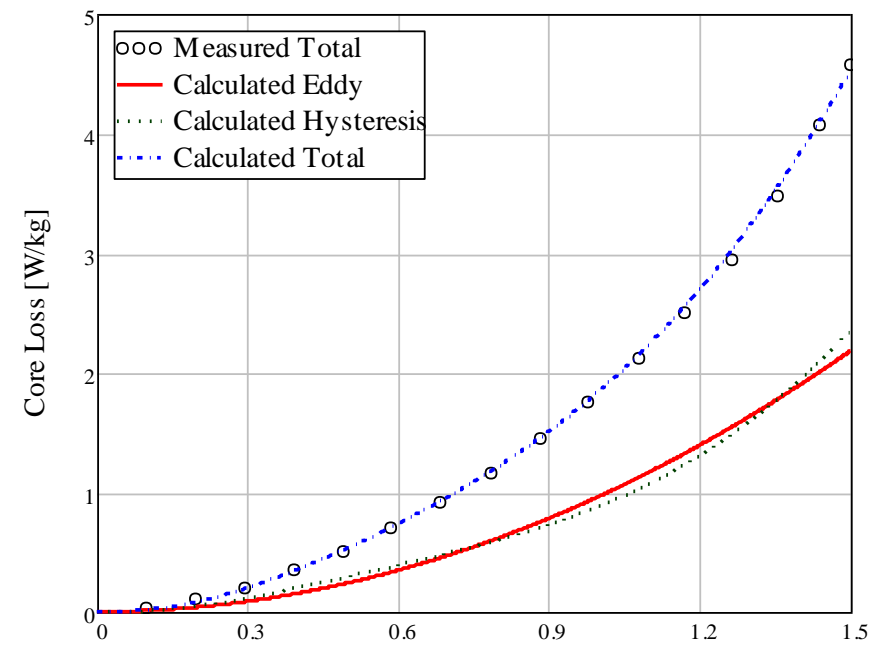

Peak Flux Density [T]

Fig. 8. Comparison of calculated and measured core losses for the flux waveform in the stator yoke at full-load.

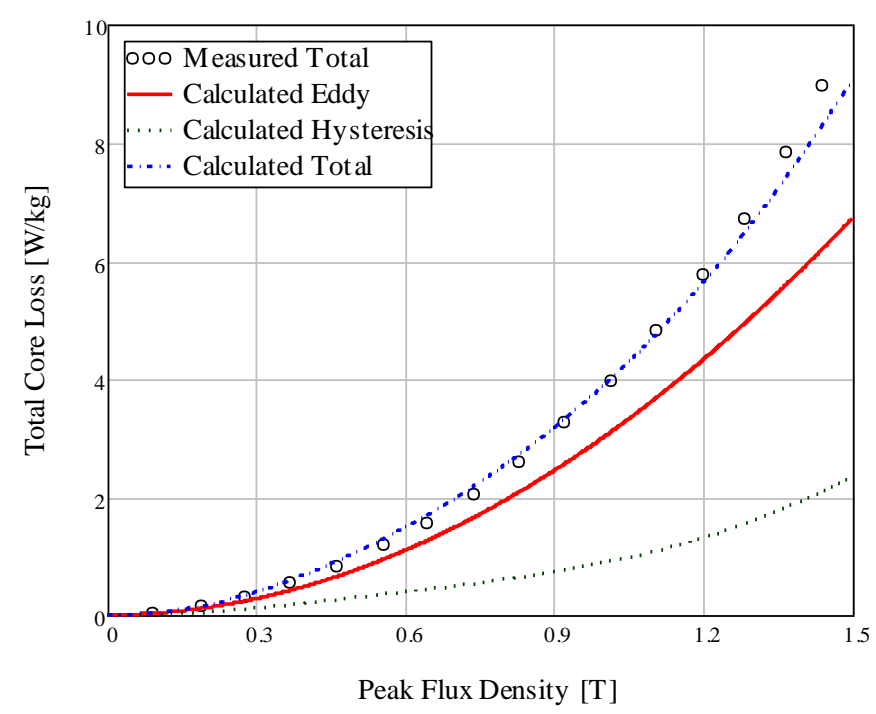

Fig. 9. Comparison of calculated and measured core losses for the flux waveform in the stator tooth at full-load. 


\section{Modeling OF MinOR Hysteresis LoOPS}

It has been shown in the previous section that core losses under arbitrary flux waveforms in the absence of minor hysteresis loops can be predicted considering the non-uniform distribution of the magnetic field inside the lamination. This approach can be adopted to calculate core losses in electrical machines, where the flux waveforms are symmetric with two flux reversals per cycle. However, the flux waveforms in many electrical machines contain significant harmonic content. Depending on the phase and magnitude of these harmonics, the resulting flux waveforms inside the machine core may contain additional flux reversals, which cause minor loops to occur inside the main hysteresis loop. These minor loops represent an additional loss component to the main loop hysteresis loss calculated by (8).

Many empirical formulas have been derived based on experimental studies to evaluate minor loop hysteresis losses [11-13]. These formulas can provide reasonable estimates of the hysteresis losses under certain conditions. However, they cannot be relied on to predict minor loop losses under a large variety of possible practical flux waveforms in electrical machines ranging from the distorted flux in induction machines operating with PWM supplies [14] to the unipolar flux in SR machine stator poles [15].

The hysteresis process is such a complex phenomenon that it is impossible to accurately predict hysteresis loss under any arbitrary flux waveform using a single empirical formula. Therefore, a hysteresis model has to be adopted in order to predict the non-linear behavior of the magnetic material under distorted excitations. Many models are available for hysteresis loop modeling, ranging from purely mathematical models [16] to physics-based models such as the Preisach model [17, 18], the Jiles-Atherton model [19], and the Energetic model [20]. Here, an Energetic model is applied to predict the static hysteresis losses for any arbitrary flux waveform.

The energetic model is suitable for fast core loss calculations in electrical machine finite element simulations as the magnetic field $H$ can be calculated directly by one equation from the flux density waveforms, which are available in the post- processing stage. The Energetic model simulation of the hysteresis loops is much faster than the Preisach model which requires simulating the magnetization processes, and even faster than the Jiles-Atherton model, where the process is also simulated by one equation, but an integral over $d H$ is required. In addition, the energetic model is capable of considering the dependence of magnetization on temperature, stress, and magnetization direction. These parameters are obtainable by means of finite element simulation, and their influence on the machine core losses can be considered by the Energetic model. This could allow accurate prediction of electrical machine efficiency under its actual operating conditions.
In the Energetic model, the magnetic field $H$ is calculated from the relative magnetization $\left(m=M / M_{s}\right)$ by,

$$
\begin{aligned}
& H=N_{e} M_{s} m+\operatorname{sgn}(m) h\left\{\left[(1+m)^{1+m}(1-m)^{1-m}\right]^{g / 2}-1\right\} \\
& +\operatorname{sgn}\left(m-m_{0}\right)\left(\frac{k}{\mu_{0} M_{s}}+C_{r} H_{r}\right) \times\left[1-\kappa \exp \left(-\frac{q}{\kappa}\left|m-m_{0}\right|\right)\right]
\end{aligned}
$$

The first term of equation (9) represents the linear material behavior with $N_{e}, M_{s}$ being the demagnetization factor and saturation magnetization. The second term represents the nolinear material behavior with $h$ and $g$ relating to saturation field and anisotropy. The third term describes the hysteresis effects, with $k$ relating to hysteresis loss, $q$ to the pinning site density, $C_{r}$ to the grain geometry and $H_{r}$ to the reversible field.

The energetic model parameters are extracted according to the procedure described in [20] using the measured static hysteresis loop at a peak flux density of $1.4 \mathrm{~T}$. Fig. 10 compares the measured hysteresis loop at $1.4 \mathrm{~T}$ with the Energetic model simulated hysteresis loop. It can be seen that the energetic model can accurately simulate the main hysteresis loop using the extracted parameters. The model simulation of minor hysteresis loops is also compared with the measured loops under the distorted flux density waveform shown in Fig. 11 at a peak flux density of $1.2 \mathrm{~T}$, which is not used in the model identification. The results displayed in Fig. 12 show good correlation between measured and simulated hysteresis loops. Table III compares the measured hysteresis energy loss under different magnitudes of in-phase third harmonic with the calculated loss by the Energetic model. The results show that the Energetic model is capable of predicting the hysteresis loss under distorted flux waveforms.

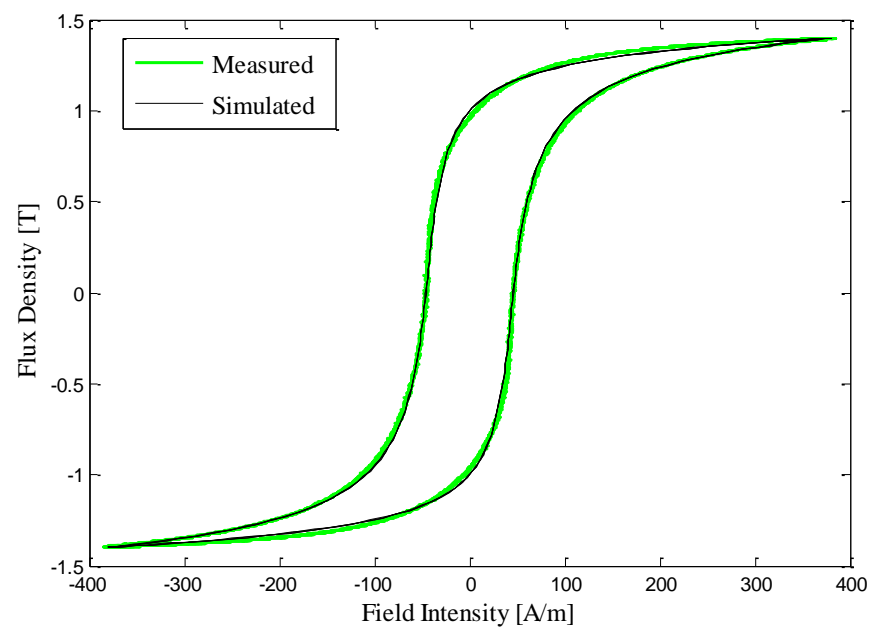

Fig. 10. Comparison between the simulated hysteresis loop by the energetic mode and the measured hysteresis loop in M45G26 lamination at $5 \mathrm{~Hz}$. 


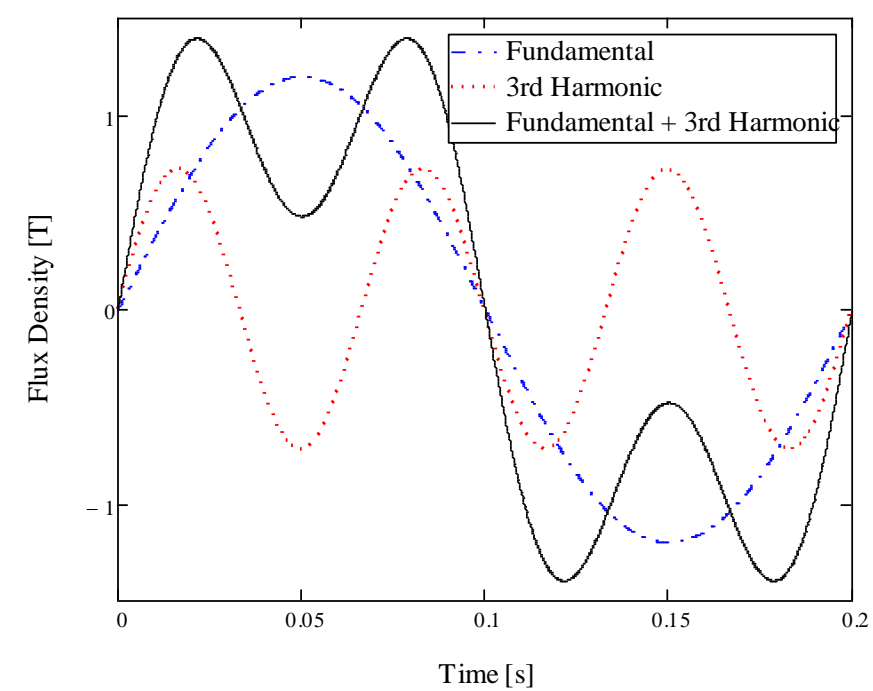

Fig. 11. Flux density waveform in the presence of $60 \%$ in-phase third harmonic.

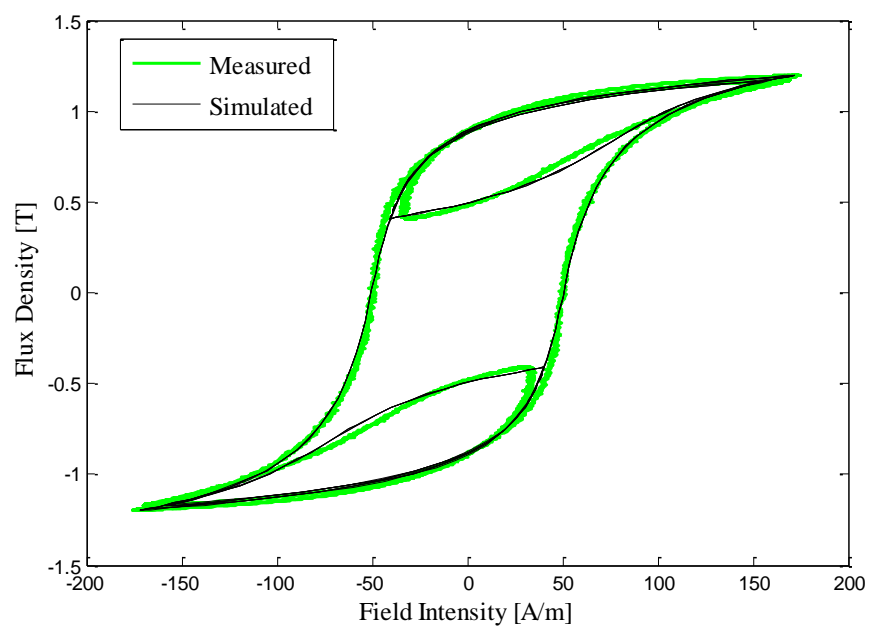

Fig. 12. Comparison of the simulated and measured hysteresis loops with third harmonic.

TABLE III

Comparison of measured and calculated hysteresis energy loss under different magnitudes of in-phase third harmonic

\begin{tabular}{|c|c|c|}
\hline$\frac{B_{3}}{B_{1}}$ & $\begin{array}{c}\text { W measured } \\
(\mathrm{J} / \mathrm{kg})\end{array}$ & $\begin{array}{c}\text { W calculated } \\
(\mathrm{J} / \mathrm{kg})\end{array}$ \\
\hline 0.2 & 0.0216 & 0.0219 \\
\hline 0.4 & 0.0267 & 0.0254 \\
\hline 0.6 & 0.0314 & 0.0308 \\
\hline 0.8 & 0.0355 & 0.0356 \\
\hline 1 & 0.0387 & 0.0393 \\
\hline
\end{tabular}

\section{CONCLUSION}

A physics-based core loss model is developed to calculate core losses considering skin effect and minor hysteresis loops. The model results are compared to the measured losses in laminations exposed to the flux waveforms in a PM machine. The results show that it is possible to calculate analytically the eddy current losses considering skin effect, even in the cases where the flux waveforms are distorted. In order to achieve accurate and computationally efficient hysteresis loss determination in the presence of minor loops, the Energetic hysteresis model is applied to calculate the quasi-static hysteresis loops. The Energetic model results are verified experimentally by comparing to the measured hysteresis loops at low frequencies.

\section{ACKNOWLEDGMENT}

This project is part of the R\&D program of the NSERC Chair entitled "Energy efficiency in electrical machines for small renewable energy production systems" established in 2009 at Concordia University. The authors acknowledge the support of the Natural Sciences \& Engineering Research Council of Canada and Hydro-Québec.

\section{REFERENCES}

[1] G. A. McCo et al, "Energy-Efficient Electric Motor Selection Handbook," Revision 3, January 1993.

[2] C. P. Steinmetz, "On the law of hysteresis (originally published in 1892)," Proc. IEEE, vol. 72, no. 2, pp. 196-221, Feb. 1984.

[3] G. Bertotti, "General properties of power losses in soft ferromagnetic materials," IEEE Trans. Magn. Vol. 24, pp. 621-630, January1988.

[4] Y. Chen and P. Pillay, "An improved formula for lamination core loss calculations in machines operating with high frequency and high flu density excitation," IEEE 37th IAS Annual Meeting, vol. 2, pp. 759766, 13-18 Oct 2002.

[5] D. M. Ionel, M. Popescu, S. J. Dellinger, et al., "on the variation with flux and frequency of core loss coefficients in electrical machine," IEEE Trans. Magn Vol. 42, No. 3, pp. 658-666, May/June 2006.

[6] A. Boglietti, A. Cavagnino, M. Lazzari, and M. Pstorelli, "Predicting iron losses in soft magnetic materials with arbitrary voltage supply: an engineering approach," IEEE Trans. Magn., vol. 39, no. 2, pp. 981 989, Mar. 2003

[7] T.L. Mthombeni and P.Pillay, "Physical basis for the variation of lamination core loss coefficients as a function of frequency and flux density," in proc. Annual Conference of the IEEE Industrial Electronics Society, pp. 1381 - 1387, Nov. 2006

[8] Y. Zhang, R. Guan, P. Pillay and M.-C. Cheng, "General core loss models on a magnetic lamination," in proc. IEEE Int. Electric Machines and Drives Conf. , 3-6 May 2009, pp. 1529 - 1534.

[9] M. Ibrahim, and P. Pillay "Novel equipment for the measurement of core losses in laminations for advanced machines," in proc. IEEE Int. Electric Machines and Drives Conf. , 15-18 May 2011, pp. 1231 - 1236.

[10] M. Manyage "Traction Motor Design," PhD Thesis, University of Cape Town, South Africa, June, 2008.

[11] T. Nakata, Y. Ishihara, and M. Nakano, "Iron losses of silicon steel core produced by distorted flux," Elect. Eng. Jpn., vol. 90, pp. 10-20, 1970.

[12] J. D. Lavers, P. P. Biringer, and H. Hollitscher, "A simple method of estimating the minor loop hysteresis loss in thin laminations," IEEE Trans. Magn., vol. 14, pp. 386-388, Sept. 1978.

[13] P. Rupanagunta, J. S. Hsu, and W. F. Weldon, "Determination of iron core losses under influence of third-harmonic flux component," IEEE Trans. Magn., vol. 27, pp. 768-777, Mar. 1991.

[14] E. Barbisio, F. Fiorillo, and C.Ragusa "Predicting loss in magnetic steels under arbitrary induction waveform and with minor hysteresis loops," IEEE Trans. Magn., vol. 40, pp. 1810 - 1819, Jul. 2004.

[15] S.D. Calverley, G.W. Jewell, R.J. Saunders, "Prediction and Measurement of Core Losses in a High-Speed Switched-Reluctance Machine," IEEE Trans. Magn., Vol. 41 , pp. 4288 - 4298, Nov. 2005.

[16] M.L. Hodgdon, "Mathematical theory and calculations of magnetic hysteresis curves", IEEE Trans. Magn. 24(6) (1988).

[17] F. Preisach, "Uber die magnetische nachwrikung," Zeitschrift fur Physik, vol. B 94, pp. 277-302, 1935.

[18] I. D. Mayergoyz, Mathematical Models of Hysteresis. New York: Springer-Verlag, 1991.

[19] D.C. Jiles, D.L. Atherton, "Theory of ferromagnetic hysteresis," $J$. Magn. Magn. Mater. 61 (1986) 48. 
[20] H. Hauser, "Energetic model of ferromagnetic hysteresis: Isotropic magnetization," J. Appl. Phys. 96, 2753 (2004).

[21] Y. Zhang, P. Pillay, M. Ibrahim, and M. Cheng "Magnetic Characteristics and Core Losses in Machine Laminations: High Frequency Loss Prediction from Low Frequency Measurements," IEEE Trans. on Industrial Applications Society, Vol. 48, No. 2, pp. 623 - 629 Mar. /Apr. 2012.

[22] L. R. Dupre, et al. "Modelling of electromagnetic phenomena in soft magnetic materials under unidirectional time periodic flux excitations", IEEE Trans. Magn., vol. 35, pp. 4171-4184, 1999.

[23] S. Zirka, et al., "Loss Separation in Nonoriented Electrical Steels", IEEE Trans. Magn., vol. 46, pp. 286-289, 2010.

[24] P. Rasilo, et al. "Model of laminated ferromagnetic cores for loss prediction in electrical machines", IET Electric Power Applications, Vol. 5, pp. 580-588, 2011.

Maged Ibrahim (M'10) received the Bachelor's degree from Alexandria University, Alexandria, Egypt in 2008, and the M.S. degree in 2011 from Concordia University, Montreal, Canada, where he is currently pursuing the $\mathrm{Ph} . \mathrm{D}$. degree in electrical engineering.

His research interests include core loss modeling in magnetic materials, design and control of electrical machines, and power electronics.

Pragasen Pillay (F'05) received the Bachelor's degree from the University of Kwa-Zulu Natal, Durban, South Africa, in 1981, the Master's degree from the University of Kwa-Zulu, Natal, Durban, South Africa, in 1983, and the Ph.D. degree from Virginia Polytechnic Institute and State University, Blacksburg, in 1987.

Currently, he is a Professor in the Department of Electrical and Computer Engineering, Concordia University, Montreal, Canada, where he holds the NSERC/Hydro Quebec Industrial Research Chair. From 1988 to 1990, he was with the University of Newcastle-upon-Tyne, Newcastle-upon-Tyne, U.K. From 1990 to 1995, he was with the University of New Orleans. From 1995 to 2007 he was with Clarkson University, Potsdam, NY, where he held the Jean Newell Distinguished Professorship in Engineering. He is also an Adjunct Professor at the University of Cape Town, Cape Town, South Africa. His research and teaching interests are in modeling, design, and control of electric motors and drives for industrial and alternate energy applications.

Dr. Pillay is a member of the IEEE Power Engineering, IEEE Industry Applications (IAS), IEEE Industrial Electronics, and IEEE Power Electronics Societies. He is a member of the Electric Machines Committee and Past Chairman of the IEEE Industrial Drives Committee of the IAS, Past Chairman of the Induction Machinery Subcommittee of the IEEE Power Engineering Society, Past Chairman of the Awards Committee of the IAS Industrial Power Conversion Department. He has organized and taught short courses in electric drives at IAS Annual Meetings. He is a Fellow of the Institution of Electrical Engineers and Technologists, U.K., and a Chartered Electrical Engineer in the U.K. He is also a Member of the Academy of Science of South Africa. He was a recipient of a Fulbright Scholarship for his Ph.D and received the Order of Mapungubwe from the President of South Africa in 2008 for contributions to South Africa in the area of energy conservation. 